\title{
On the Free Oscillation of Water in a Lake of Elliptic Boundary with Small Eccentricity and of Uniform Depth*
}

\author{
Yoshio SHIBAOKA** and Masaki TAKASHIMA **
}

\begin{abstract}
The fundamental normal mode of the free oscillation of water in a lake of elliptic boundary with small eccentricity and of uniform depth is investigated by means of a kind of boundary perturbation method. An approximate formula for the relationship between the frequency of the fundamental normal mode of oscillation and the eccentricity of the elliptic boundary of the lake is derived correct to the sixth power of the latter, which may be adequately used in case when the eccentricity is not so large.
\end{abstract}

\section{Introduction}

The problem of the free oscillations of water in an elliptic lake of uniform depth has been studied mathematically in detail by JEFFREYS and GOLDSTEIN. More recently, Professor HIDAKA has given very interesting results by means of numerical integration not only for the case of an elliptic lake of uniform depth, but also for the cases of lakes of boundaries of various other shapes and of various laws of depth. Though an exact solution expressed in terms of Mathieu functions is mathematically elegant, it is rather complicated for a numerical treatment.

It seems that there are many lakes whose boundaries can be approximated by nearly circular ellipses. In the present paper, the fundamental normal mode of the free oscillation of water in a lake of elliptic boundary with small eccentricity and of uniform depth is discussed by means of a kind of boundary perturbation method. After rather lengthy and tedious calculations an approximate formula for the relationship between the frequency of the fundamental normal mode of oscillation and the eccentricity of the elliptic boundary of the lake is derived correct to the sixth power of the latter, which may be adequately used in case when the eccentricity is not so large. While the results obtained by numerical method can be used only for a fixed value of eccentricity, our result can be useful for any value of

* Received Dec. 10, 1962

** Institute of Polytechnics, Osaka City University eccentricity so long as the eccentricity is not so large.

The similar problem has been treated by TUBOI in the same way as Lord RAYLEIGH applied to the vibration of a nearly circular membrane, but it seems that the coefficients of the terms of order higher than the fourth in $e$ cannot be obtained correctly by such method.

On the other hand, it is also shown that our result agrees exactly with that derived from an exact solution.

\section{Fundamental equation and its solution}

Let $w$ be the vertical displacement of the free surface of the lake from its equilibrium position, and let the rectangular coordinate-axes $(x, y)$ be taken in the plane of the undisturbed level as shown in Fig. 1. Then, as is well known, $w$ satisfies the equation:

$$
\frac{\partial^{2} w}{\partial t^{2}}=c^{2}\left(\frac{\partial^{2} w}{\partial x^{2}}+\frac{\partial^{2} w}{\partial y^{2}}\right),
$$

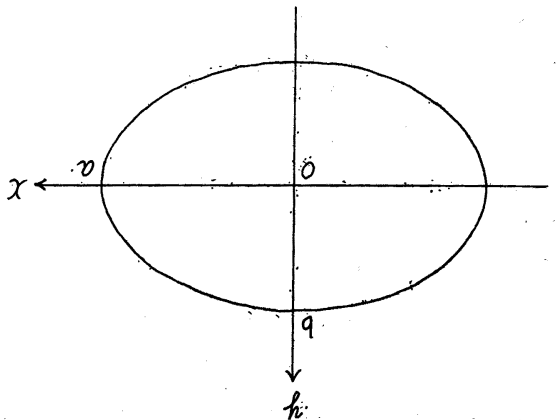

Fig. 1. 
where $c=\sqrt{g h}, g$ and $h$ being respectively the acceleration due to gravity and the uniform depth of the lake.

When the free surface of the lake oscillates in a normal mode, the displacement is of the form

$$
w=\zeta \cos \sigma t,
$$

where $\zeta$ is a function of $x, y$, and $\sigma$ is the circular frequency. If we insert (2) into (1) and write

$$
k=\frac{\sigma}{c},
$$

for simplicity, we obtain a partial differential equation for $\zeta$, namely:

$$
\frac{\partial^{2 \zeta}}{\partial x^{2}}+\frac{\partial^{2} \zeta}{\partial y^{2}}+k^{2} \zeta=0
$$

Since the boundary of the lake is vertical everywhere, the boundary condition is given by

$$
\left(\frac{\partial \zeta}{\partial n}\right) \text { on the ellipse }=0
$$

where $\frac{\partial}{\partial n}$ indicates the differentiation along the normal to the vertical bounding wall.

We now introduce the polar coordinates $(r$, $\theta$ ) with the center of the elliptic lake as origin and with the major axis of the ellipse as initial line. Then, Equations (4) and (5) become respectively

$$
\begin{gathered}
\frac{\partial^{2} \zeta}{\partial r^{2}}+\frac{1}{r} \frac{\partial \zeta}{\partial r}+\frac{1}{r^{2}} \frac{\partial^{2} \zeta}{\partial \theta^{2}}+k^{2} \zeta=0 \\
\left(r^{2} \frac{\partial \zeta}{\partial r}-\frac{d r}{d \theta} \frac{\partial \zeta}{\partial \theta}\right) \text { on the ellipse }=0 .
\end{gathered}
$$

If we restrict our attention to those normal modes of oscillation in which the displacement is symmetrical about both the axes of the ellipse, as in the case of the fundamental normal mode under consideration, the appropriate particular solutions of Equation (6) can be expressed in terms of Bessel functions and circular functions as

$$
\begin{aligned}
& \zeta=J_{\mathbf{2}}(k r) \cos 2 m \theta \\
& \quad(m=0,1,2,3 \cdots \cdots)
\end{aligned}
$$

The complete expression for $\zeta$ in the present case is obtained by combining the particular solutions embodied in (8) with all admissible values of $m$, viz.

$$
\begin{aligned}
\zeta & =\sum_{m=0}^{\infty} A_{2 m} J_{2 m}(k r) \cos 2 m \theta \\
& =A_{o} J_{0}(k r)+\sum_{m=1}^{\infty} A_{2 m} J_{2 m}(k r) \cos 2 m \theta,
\end{aligned}
$$

where $A_{0}$ and $A_{2 m}(m=1,2,3 \cdots \cdots)$ are arbitrary constants. The first term on the right-hand side of (9) corresponds to the fundamental normal mode of oscillation of water in an exactly circular lake and " $\Sigma$ " part signifies the perturbation terms.

We have now to find the form of the equation representing the boundary. Since the ellipse under consideration is nearly circular, the radius vector is nearly constant. Accordingly, we may take

$$
r=a+\ddot{o r},
$$

$\delta r$ being a small function of $\theta$. It is easily found that $\delta r$ has the form of

$$
\begin{aligned}
\delta r= & r-a \\
= & a\left\{\left(-\frac{1}{4} e^{2}-\frac{7}{64} e^{4}-\frac{17}{256} e^{6}\right)\right. \\
& +\left(\frac{1}{4} e^{2}+\frac{1}{16} e^{4}+\frac{11}{512} e^{6}\right) \cos 2 \theta \\
& \left.+\left(\frac{3}{64} e^{4}+\frac{9}{256} e^{6}\right) \cos 4 \theta+\frac{5}{512} e^{6} \cos 6 \theta\right\},
\end{aligned}
$$

where $e$ is the eccentricity of the elliptic boundary of the lake and all the terms of order higher than the sixth in $e$ are neglected.

Rewriting (7) by using (10), we obtain

$$
\left(r^{2} \frac{\partial \zeta}{\partial r}-\frac{d r}{d \theta} \frac{\partial \zeta}{\partial \theta}\right)_{\gamma=a+\delta r}=0
$$

3. Determination of the frequency of the fundamental normal mode of oscillation

Substituting from (9) into (12), we obtain $k\left\{a^{2}+2 a(\delta r)+(\delta r)^{2}\right\}$

$$
\begin{aligned}
& \times\left[A_{0} J_{0}^{\prime}\{k(a+\delta r)\}\right. \\
& \left.+\sum_{m=1}^{\infty} A_{2 m} J_{2 m^{\prime}}\{k(a+\delta r)\} \cos 2 m \theta\right] \\
& +\frac{d}{d \theta}(\delta r) \sum_{m=1}^{\infty} 2 m A_{2 m} J_{2 m}\{k(a+\delta r)\} \sin 2 m \theta=0 .
\end{aligned}
$$


If we divide both sides by $A_{0}$ and expand Bessel functions in power series of $(k \delta r),(13)$ becomes

$$
\begin{aligned}
& k\left\{a^{2}+2 a(\delta r)+(\delta r)^{2}\right\} \\
& \times\left[J_{0}^{\prime}(k a)+J_{0}^{\prime \prime}(k a)(k \delta r)+\frac{1}{2} J_{0}^{\prime \prime \prime}(k a)(k \delta r)^{2}\right. \\
& +\frac{1}{6} J_{0}^{\prime \prime \prime \prime}(k a)(k \delta r)^{3}+\cdots \cdots+\sum_{m=1}^{\infty} \frac{A_{2 m}}{A_{0}}\left\{J_{2 m^{\prime}}(k a)\right. \\
& +J_{2 m^{\prime \prime}}(k a)(k \delta r)+\frac{1}{2} J_{2 m^{\prime \prime \prime}}(k a)(k \delta r)^{2} \\
& +\cdots \cdots\} \cos 2 m \theta]+\frac{d}{d \theta}(\delta r) \sum_{m=1}^{\infty} 2 m \frac{A_{2 m}}{A_{0}}\left\{J_{2 m}(k a)\right. \\
& \left.+J_{2 m^{\prime}}(k a)(k \delta r)+\cdots \cdots\right\} \sin 2 m \theta=0 .
\end{aligned}
$$

Since $\delta r$ depends on $e^{2}$ as may be seen from (11), both $k$ and $\frac{A_{2 m}}{A_{0}}$ involved in (14) will also be expanded in power series of $e^{2}$. Hence we may assume

$$
k a=u_{0}+u_{1} e^{2}+u_{2} e^{4}+u_{3} e^{6}+\cdots \cdots .
$$

$A_{2 m}(m=1,2,3, \cdots \cdots)$ are all small relatively to $A_{0}$, since the type of oscillation can only differ a little from what it would be, were the boundry an exact circle. Hence $\frac{A_{2 m}}{A_{0}}$ may be considered to be of order $e^{2}$. Accordingly, we may assume

$$
\begin{array}{r}
\frac{A_{2 m}}{A_{0}}=P_{2 m} e^{2}+Q_{2 m} e^{4}+R_{2 m} e^{6}+\cdots \cdots \\
(m=1,2,3, \cdots \cdots)
\end{array}
$$

By (15), (16) and the expression (11) for $\delta r$, the left-hand side of (14) is expanded in a power series of $e^{2}$. If we neglect all the terms of order higher than the sixth in $e$, we obtain sixteen equations from the condition that (14) must hold independently of the values of both $e$ and $\theta$.

By solving these equations, we can determine all the required coefficients in (15) and (16). Namely, $u_{o}$ is given by the smallest positive root of the next equation:

$$
J_{o}^{\prime}(u)=0 \text { or } J_{1}(u)=0 .
$$

By McMAHON, such a root is

$$
u_{0}=3.8318 \text {, }
$$

this being the value of $k a$ for the fundamental normal mode of oscillation of water in an exactly circular lake of radius $a$.

If we simplify the other coefficients determined from the foregoing equations by using the recurrence formulae for Bessel functions, it is found that they are as follows:

$$
\begin{aligned}
& {\left[\begin{array}{l}
u_{1}=\frac{1}{4} u_{o}=0.9580, \\
u_{2}=\frac{5}{32} u_{o}+\frac{1}{64} u_{0}^{3}=1.4778, \\
u_{3}=\frac{15}{128} u_{o}+\frac{5}{256} u_{o}^{3}=1.5479,
\end{array}\right]} \\
& {\left[\begin{array}{l}
P_{2}=\frac{1}{8} u_{0}^{2}, \\
P_{2 m}=0,(m=2,3,4 \cdots \cdots)
\end{array}\right]} \\
& {\left[\begin{array}{l}
Q_{2}=\frac{1}{16} u_{0}^{2}, \\
Q_{4}=\frac{1}{512} \cdot \frac{u_{0}{ }^{6}}{12-u_{0}^{2}}, \\
Q_{2 m}=0,(m=3,4,5, \cdots \cdots)
\end{array}\right]} \\
& R_{2 m}=0 .(m=4,5,6 \cdots \cdots)
\end{aligned}
$$

In order to determine $R_{2}, R_{4}$ and $R_{6}$, we need considerably laborious calculations. Fortunately, however, they are unnecessary in the present case when our aim is to determine $u_{o}$, $u_{1}, u_{2}$ and $u_{3}$.

By (3), (15), (18) and (19), we obtain

$$
\begin{gathered}
\frac{\sigma}{c} a=u_{o}+\frac{1}{4} u_{o} e^{2}+\left(\frac{5}{32} u_{o}+\frac{1}{64} u_{o}^{3}\right) e^{4} \\
+\left(\frac{5}{128} u_{o}+\frac{5}{256} u_{o}^{3}\right) e^{6}+0\left[e^{8}\right] \\
=3.8318+0.9580 e^{2}+1.4778 e^{4} \\
+1.5479 e^{6}+0\left[e^{8}\right] .
\end{gathered}
$$

This is an approximate formula for the relationship between the circular frequency of the fundamental normal mode of oscillation and the eccentricity of the elliptic lake, which is correct to the sixth power of the eccentricity. This relationship is also shown graphically in Fig. 2. 


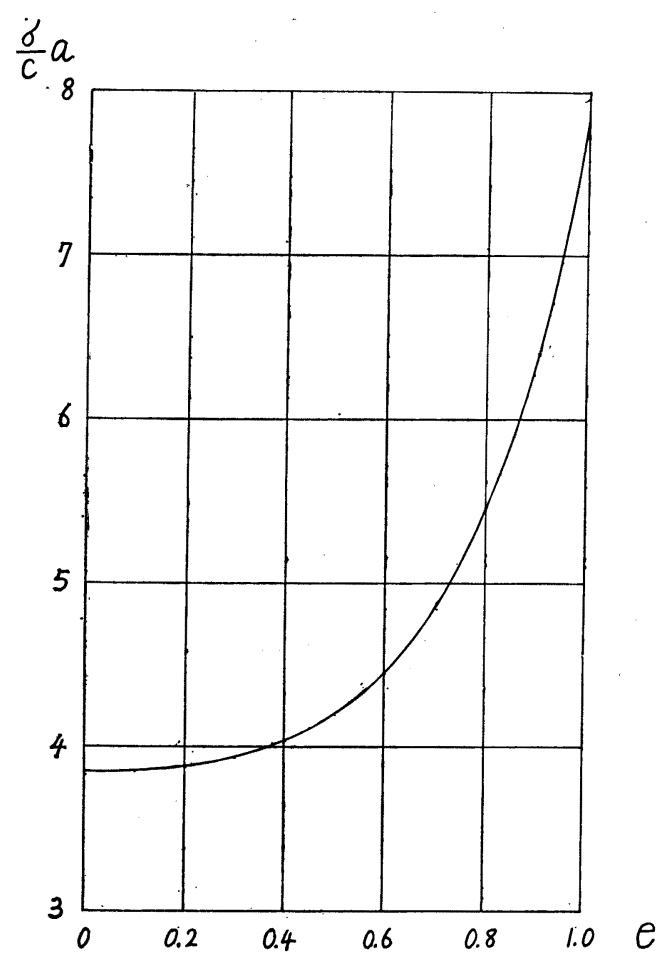

Fig. 2, The relation between the circular frequency of the fundamental normal mode of the free oscillation and the eccentricity of the elliptic boundary of the lake.

\section{Derivation from an exact solution}

Denoting the focal length of the ellipse by $2 h$, we now introduce the elliptic coordidates $(\xi, \eta)$ defined as:

$$
x+i y=h \cosh (\xi+i \eta) .
$$

Then, Equation (4) and boundary condition (5) become respectively

$$
\begin{gathered}
\frac{\partial^{2} \zeta}{\partial \xi^{2}}+\frac{\partial^{2 \zeta}}{\partial \eta^{2}}+2 k_{1}^{2}(\cosh 2 \xi-\cos 2 \eta) \zeta=0 \\
\left(\frac{\partial \zeta}{\partial \xi}\right)_{\xi=\xi_{0}}=0
\end{gathered}
$$

where

$$
2 k_{1}=k h=\frac{\sigma}{c} h,
$$

and $\xi=\xi_{o}$ signifies the boundary of the ellipse.

If we restrict our attention to those normal modes of oscillation in which the displacement is symmetrical about both the axes of the ellipse as before, the appropriate particular solution of Equation (25) can be written

$$
\zeta=C e_{o}(\xi, q) c e_{o}(\eta, q),
$$

where $c e_{o}(\eta, q)$ and $C e_{o}(\xi, q)$ are Mathieu and modified Mathieu functions of zeroth order respectively, and

$$
q=k_{1}^{2}=\frac{1}{4} k^{2} h^{2}=\frac{1}{4} \frac{\sigma^{2}}{c^{2}} h^{2} .
$$

Substituting from (28) into (26), we obtain

$$
C e_{o}{ }^{\prime}\left(\xi_{o}, q\right)=0 \text {. }
$$

If we use the expanding formula for $C e_{o}(\xi, q)$ :

$$
\begin{aligned}
& C e_{o}(\xi, q) \\
= & \frac{c e_{o}\left(\frac{\pi}{2}, q\right)}{A_{o}^{(o)}} \sum_{r=0}^{\infty}(-1)^{r} A_{2 r}^{(o)} J_{2 r}\left(2 k_{1} \cosh \xi\right),
\end{aligned}
$$

(30) leads to

$$
\sum_{r=0}^{\infty}(-1)^{r} A_{2 r^{(o)}} J_{2 r^{\prime}}\left(2 k_{1} \cosh \xi_{o}\right)=0 .
$$

Since

$$
\begin{aligned}
& k_{1}=\frac{1}{2} k h, \\
& h=\sqrt{a^{2}-b^{2}}=a e,
\end{aligned}
$$

and

$$
\cosh \xi_{o}=\frac{a}{\sqrt{a^{2}-b^{2}}}=\frac{1}{e},
$$

we find

$$
2 k_{1} \cosh \xi_{o}=k a,
$$

which gives

$$
\sum_{r=0}^{\infty}(-1)^{r} A_{2 r^{(o)}} J_{2 r^{\prime}}(k a)=0 .
$$

Now, let us suppose that we may take

$$
k a=u_{0}+u_{1} e^{2}+u_{2} e^{4}+u_{3} e^{6}+\cdots \cdots,
$$

as before, and let us use the following relations

$$
\left\{\begin{array}{l}
A_{2}{ }^{(0)}=\left\{-\frac{1}{8}(k a)^{2} e^{2}+\frac{7}{8192}(k a)^{6} e^{6}+\cdots\right\} A_{o}{ }^{(o)} \\
A_{4}{ }^{(0)}=\left\{\frac{1}{512}(k a)^{4}+\cdots \cdots\right\} A_{o}^{(o)}, \\
A_{6}{ }^{(0)}=\left\{-\frac{1}{73728}(k a)^{6} e^{6}+\cdots \cdots\right\} A_{o}^{(o)},
\end{array}\right\}
$$



and of Uniform Depth

Then, the left-hand side of (34) can be expanded in a power series of $e^{2}$. If we neglect the terms of order higher than the sixth in $e$, we can determine $u_{0}, u_{1}, u_{2}$ and $u_{3}$ from the condition that (34) must hold independently of the value of $e$. The result obtained in such a way agrees exactly with the preceding one.

\section{Summary}

The fundamental normal mode of the free oscillation of water in a lake of elliptic boundary with small eccentricity and of uniform depth is investigated by means of a kind of boundary perturbation method. Carrying out rather lengthy and tedious calculations, an approximate formula for the relationship between $\frac{\sigma}{c} a$ and $e$ is derived correct to the sixth power of $e$, where $\sigma$ is the circular frequency of the fundamental normal mode of oscillation, $a$ a half-length of the major axis of the ellipse, $e$ the eccentricity, and $c=\sqrt{g h}, g$ and $h$ being respectively the acceleration due to gravity and the uniform depth of the lake.

This result may be particularly useful for calculating the frequency or the period of the fundamental normal mode of oscillation in case when an elliptic lake differs only slightly from a circular lake. It is readily shown that the frequency (period) of the fundamental normal mode of oscillation of water in an elliptic lake with small eccentricity is higher (less) than that of an exactly circular lake of the same area as the former.
The boundary perturbation method presented in this paper may be applied not only to an elliptic lake, but also to any lake having nearly circular boundary and a small parameter like an eccentricity in case of an ellipse.

\section{Acknowledgements}

The writers wish to express their cordial thanks to Professor Y.SAITO of Osaka City University for his many valuable advices throughout this work. Their thanks are also due to Professor T. KAWAMURA of Nara Women's University for his kind inspection of the manuscript.

\section{References}

Goldstein, S. (1927): The free oscillations of water in a canal of elliptic plan. Proc. Lond. Math. Soc., 28, 91-101.

HIDAKA, K. (1931): The oscillations of water in spindle-shaped and elliptic basins as well as the associated problems. (Problems of water oscillations in various types of basins and canals.Part I) Mem. Imp. Mar. Obs. (Kobe, Japan), 4, 99-219.

JEFFREYS, H. (1924): The free oscillations of water in an elliptical lake. Proc. Lond. Math. Soc., 23, 455-476.

McMahon, J. (1894-95): On the roots of the Bessel and certain related functions. Ann. Math., 9, 23-30.

Rayleigh, J. W. S.: The Theory of Sound. Vol. I, 2nd ed., 336-343.

TUвоI, I. (1935): Free oscillations in a lake having non-elongated and smooth boundary. Umi to Sora., 15, 189-194. (in Japanese) 